\section{MS23-P2 Local structure of semicrystalline P3HT films probed by nanofocused diffraction}

Ruslan Kurta ${ }^{1,2}$, Linda Grodd ${ }^{3}$, Eduard Mikayelyan ${ }^{3}$, Oleg Gorobtsov $^{2,4}$, Ivan Zaluzhnyy ${ }^{2,5}$, Ilaria Fratoddi ${ }^{6,7}$, Iole Venditti ${ }^{7}$, Maria Vittoria Russo ${ }^{7}$, Michael Sprung ${ }^{2}$, Souren Grigorian ${ }^{3}$, Ivan Vartanyants ${ }^{2,5}$

1. European XFEL GmbH, Albert-Einstein-Ring 19, D-22761 Hamburg, Germany

2. Deutsches Elektronen-Synchrotron DESY, Notkestraße 85, D-22607 Hamburg, Germany

3. Department of Physics, University of Siegen, Walter-Flex-Straße 3, D-57072 Siegen, Germany

4. National Research Center "Kurchatov Institute", Kurchatov Square 1, 123182 Moscow, Russia

5. National Research Nuclear University, "MEPhI", 115409 Moscow, Russia

6. CNIS, University of Rome Sapienza, P.le A. Moro 5, I-00185 Rome, Italy

7. Department of Chemistry, University of Rome Sapienza, P.le A. Moro 5, I-00185 Rome, Italy

email: ruslan.kurta@xfel.eu

Semicrystalline conjugated polymers are promising cost-effective candidates for organic electronic devices, in particular, for organic field-effect transistors and solar cells [1]. One of the most studied compounds is poly(3-hexylthiophene) (P3HT) and its blends. Typical feature of this polymer is a mixture of poor and well organized domains, the latter are addressed to be crystalline and assumed to strongly improve device performance [2]. Our study aims to improve understanding of the role of nanoparticle additives in the structure formation and nanomorphology of P3HT in order to enhance functional properties of optoelectronic devices.

We present results of nanofocused x-ray studies of structural properties of P3HT blends with gold nanoparticles (AuNPs) [3-5]. The hidden structural features of semicrystalline polymer films are revealed by applying the novel $\mathrm{X}$-ray cross-correlation analysis (XCCA) technique [6-8]. In comparison with radial intensity curves usually analyzed in scattering experiments, the Fourier spectra of the cross-correlation functions provide extended information about the structural order in the system. Spatially resolved maps of orientational distribution of crystalline domains allow us to distinguish sample regions of predominant face-on morphology, with a continuous transition to edge-on morphology. As compared to a pristine P3HT film, the P3HT/AuNPs blend is characterized by substantial ordering of crystalline domains, which can be induced by $\mathrm{Au}$ nanoparticles. We also observed stronger structural variations of the P3HT matrix in the film regions with higher concentration of gold nanoparticles.

[1] Salleo A, Kline R J, Delongchamp D M, Chabinyc M L (2010). Adv. Mater. 22, 3812.

[2] Sirringhaus H et al. (1999). Nature 401, 685.

[3] Kurta R P et al. (2014). J. Phys.: Conf. Series 499, 012021.

[4] Quintiliani M et al. (2014). J. Mater. Chem. C 2, 2517.

[5] Kurta R P et al. (2014). Phys. Chem. Chem. Phys. 17,7404 .
[6] Wochner P et al. (2009). Proc. Natl. Acad. Sci. USA 106, 11511.

[7] Altarelli M, Kurta R P, Vartanyants I A (2010). Phys. Rev. B 82, 104207.

[8] Kurta R P, Altarelli M, Vartanyants I A (2013). Adv. Cond. Matt. Phys. 2013, 959835.

Keywords: Semicrystalline polymers, nanofocused diffraction, $\mathrm{x}$-ray cross-correlation analysis 